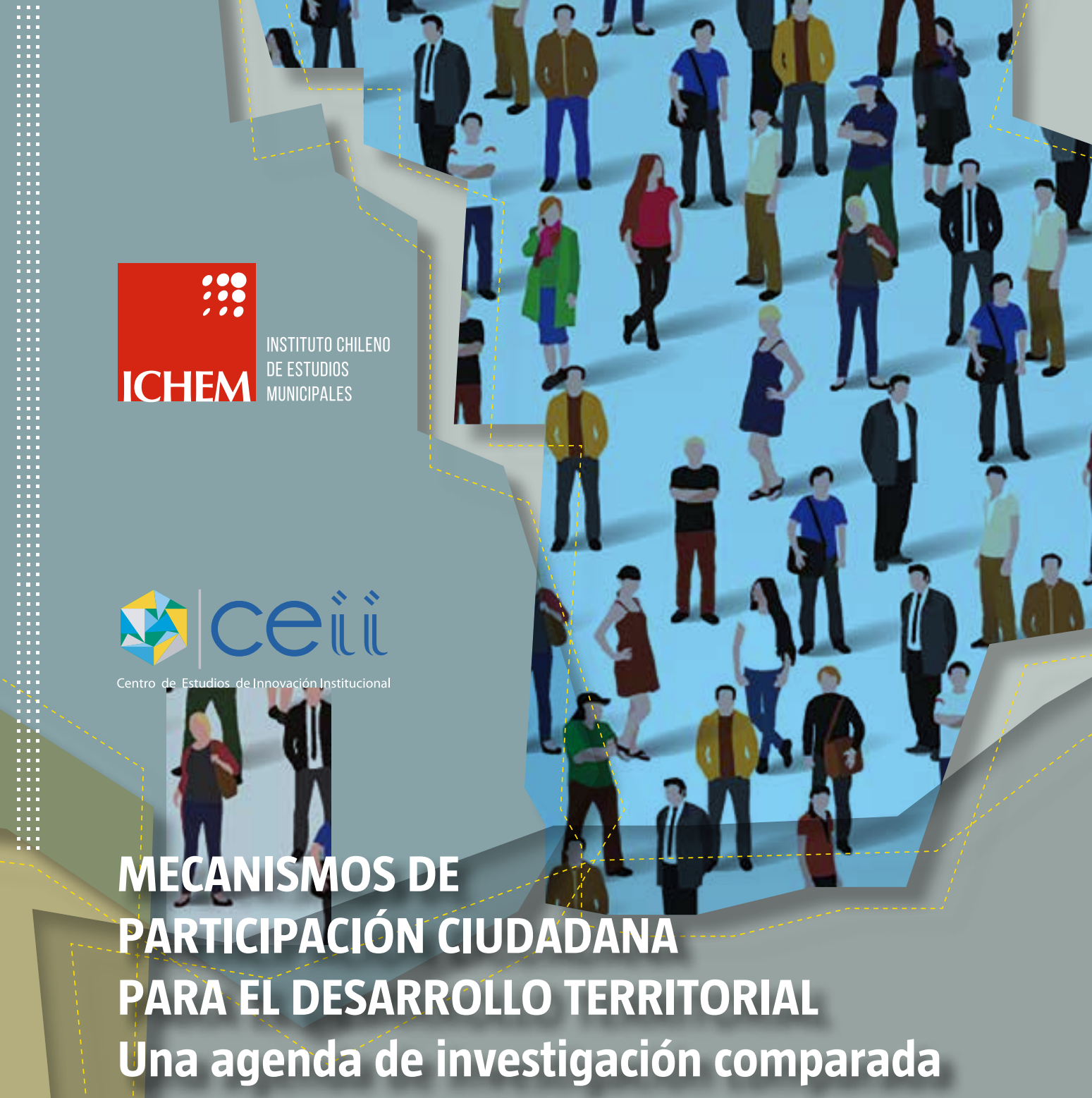




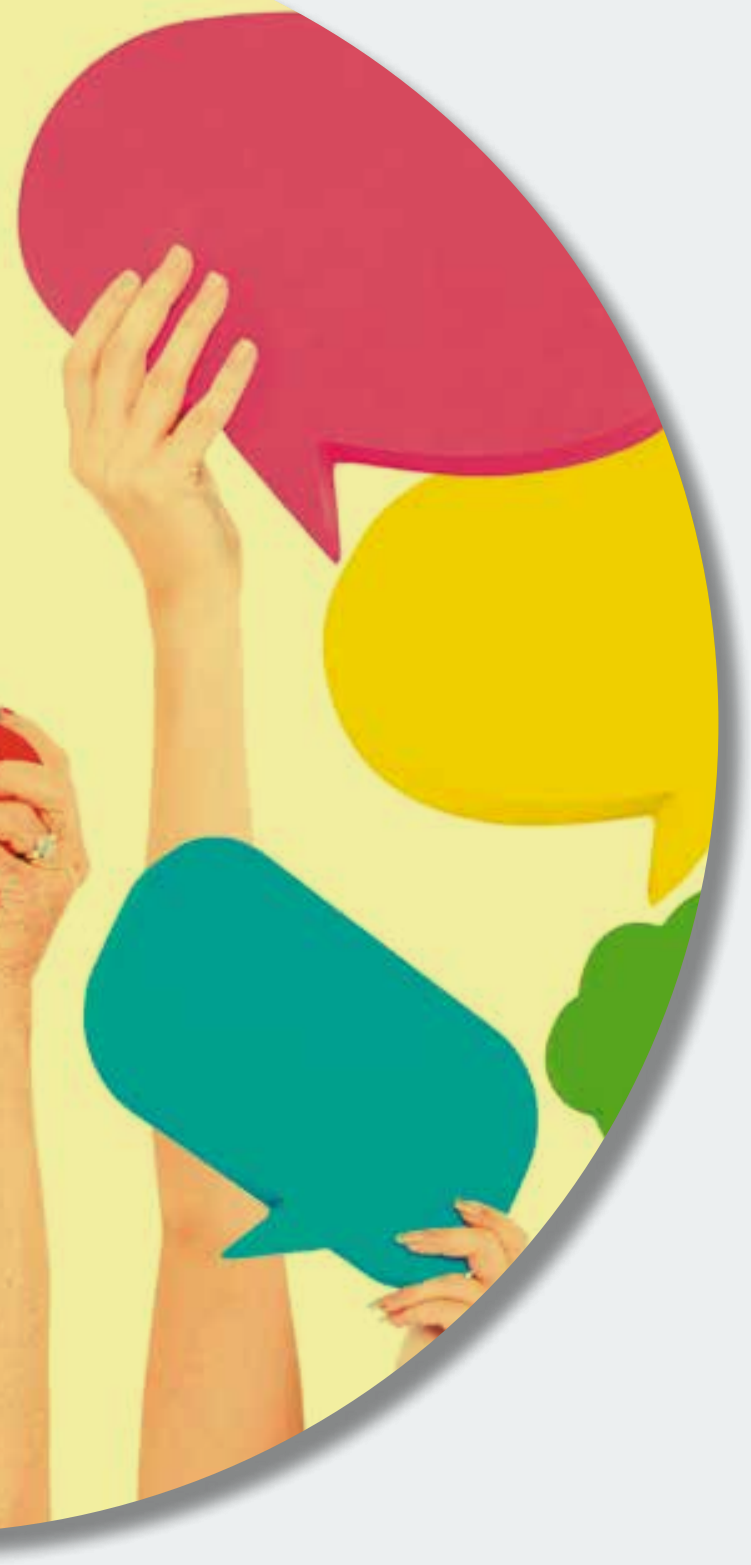

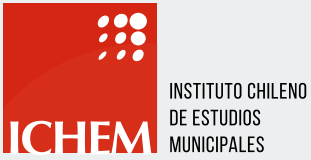

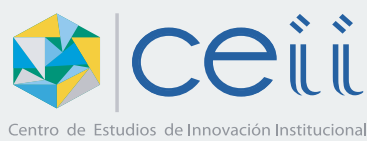

\section{MECANISMOS DE PARTICIPACIÓN CIUDADANA \\ PARA EL DESARROLLO TERRITORIAL. \\ UNA AGENDA DE INVESTIGACIÓN COMPARADA}

Dra. Melina Guardamagna | Dr. José Hernández B.

Primera edición: mayo, 2020.

\section{(c) Universidad Autónoma de Chile}

Centro de Comunicación de las Ciencias

http://ciencias.uautonoma.cl

Santiago, Chile

Instituto Chileno de Estudios Municipales (ICHEM)

https://ichem.uautonoma.cl

\section{Universidad Nacional de Cuyo}

Centro de Estudios de Innovación Institucional (CEII)

Mendoza, Argentina

https://ceii.com.ar

\section{Registro de propiedad intelectual N. ${ }^{\circ} 2020-A-3282$}

\section{Agradecimientos}

Los autores agradecen el trabajo realizado por Pablo Díaz, del equipo del CEIl de la Universidad Nacional de Cuyo, Argentina, y por Alondra Arellano, del ICHEM, para completar el presente estudio.

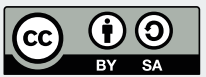

Este material puede ser copiado y redistribuido por cualquier medio o formato, además se puede remezclar, transformar y crear a partir del material siempre y cuando se reconozca adecuadamente la autoría y las contribuciones se difundan bajo la misma licencia del material original.
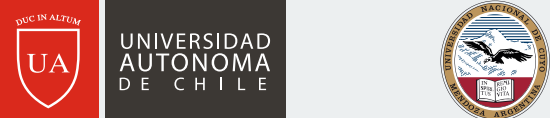

UNCUYO UNIVERSIDAD NACIONAL DE CUYO 
ÍNDICE

$\rightarrow$ Introducción

2

> Desarrollo territorial y participación ciudadana

3

$\rightarrow$ Los casos de Argentina y Chile

4

$\rightarrow$ Conclusiones

14

> Bibliografía

16

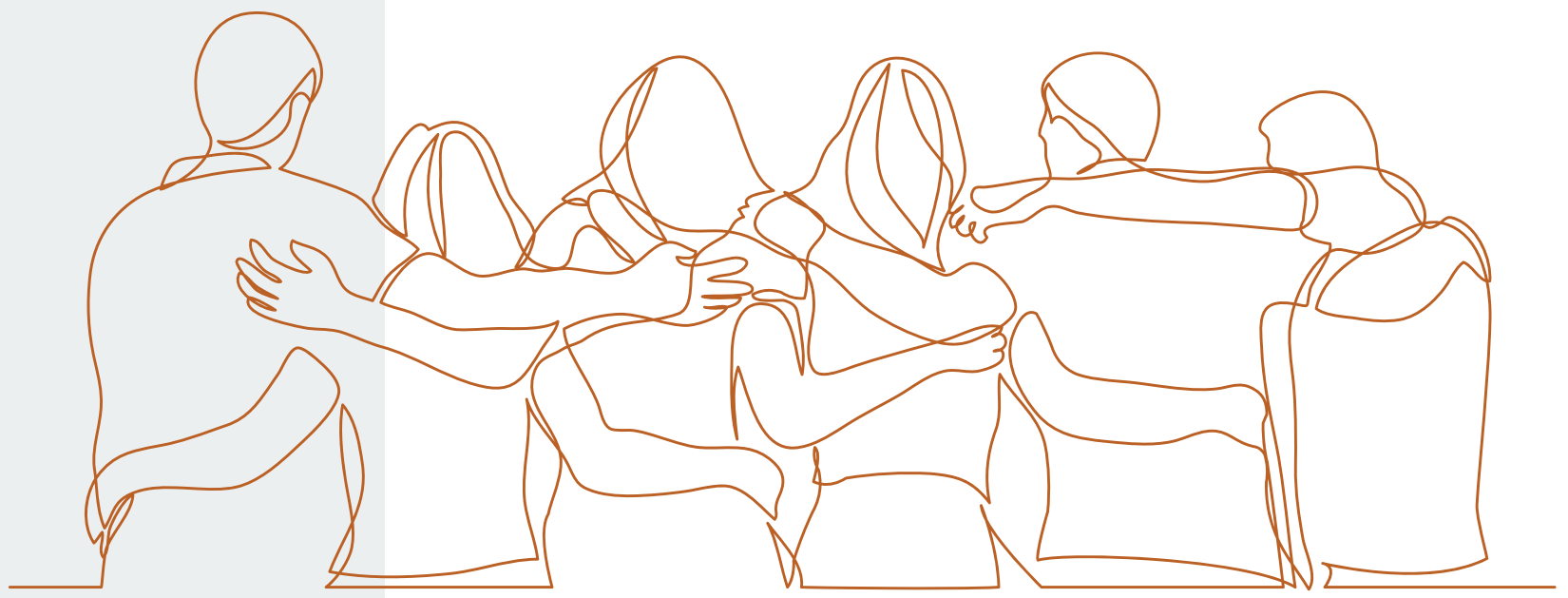




\section{INTRODUCCIÓN $\rightarrow$}

Desde el retorno a la democracia, América Latina ha sido escenario de variadas reformas institucionales que han buscado, de diversas maneras, acercar la acción estatal a la ciudadanía. Dichos esfuerzos, basados muchas veces en mecanismos institucionales de participación, se enfocaron en una doble meta: por una parte, profundizar y consolidar los valores democráticos a través del involucramiento ciudadano en la vida pública; y por la otra, el mejoramiento de la acción estatal, al contar, las administraciones públicas, con mayor y mejor información para la toma de decisiones (Harrison y Sayogo, 2014; Evans y Campos, 2012).

Aun así, los resultados de este tipo de estrategias han sido diversos y las perspectivas de la participación ciudadana están llenas de luces y sombras. Hay quienes abogan por una mayor participación y por la superación de la democracia representativa por una participativa (De Sousa Santos, 2003), y están quie- nes señalan los problemas de fondo que implica la participación ciudadana en la gestión (Patsias et. al., 2013; Andrews et. al., 2008; Irvin y Stanbury, 2004).

Con el ánimo de realizar un primer acercamiento a los mecanismos de participación ciudadana en un sector específico -el ordenamiento territorial-, este estudio busca presentar una breve comparación entre dos países con diseños institucionales casi antagónicos, de manera que podamos definir si la centralización o descentralización de competencias puede llegar a impactar en el diseño, implementación y evaluación de los mecanismos de participación.

Este estudio es parte de un proyecto de investigación interuniversitario entre el Centro de Estudios de Innovación Institucional (CEII) de la Universidad Nacional de Cuyo (Argentina) y el Instituto Chileno de Estudios Municipales (ICHEM) de la Universidad Autónoma de Chile. 


\section{DESARROLLO TERRITORIAL Y PARTICIPACIÓN CIUDADANA}

El objetivo del trabajo es aportar a la reflexión teórica y metodológica en torno a la implementación de mecanismos de participación ciudadana en políticas públicas destinadas al desarrollo del territorio, entendiendo que no es posible pensar en este sin involucramiento ciudadano. Para la teoría del desarrollo endógeno, la participación está indisolublemente ligada a él, ya que la comunidad local debe ser parte del proceso para garantizar el éxito y la sustentabilidad. De esta forma, desarrollo territorial y participación son entendidos como dos ejes inseparables (Pragier, 2009).

Sin embargo, así como la participación aparece como un componente central, también constituye un elemento controvertido de las políticas públicas destinadas al desarrollo del territorio. Esto es así porque, como sostiene Manero, se ponen en juego «nuevas premisas en las formas de gestión, en la búsqueda de opciones basadas en la voluntad de acuerdo, en el riesgo compartido y en la aparición de posturas fa- vorables a la transparencia de las decisiones» (2010: 49), lo que implica, siguiendo a Zoido (2007), una nueva cultura política con ciudadanos más activos, organizados y conscientes de los problemas que afectan su entorno de vida.

Las políticas de desarrollo territorial, en consecuencia, son intrínsecamente participativas porque tienen que ver con la conformación social, económica y política de los territorios. El desarrollo territorial, en este sentido, se define como un proceso de transformación. A través de la organización y la dinamización del territorio y de la puesta en marcha de metodologías dinámicas, flexibles y-agregamos nosotrosparticipativas para la organización social, pretende: alcanzar un alto grado de innovación y crecimiento económico sustentable y lograr altos niveles de capital social y cultural, reduciendo los índices de pobreza y fomentando la inclusión y el mejoramiento de la calidad de vida (Sili, 2005; Alburquerque, 2004; Schejtman, 2004). 


\section{$\rightarrow$ LOS CASOS DE ARGENTINA Y CHILE}

Existen grandes diferencias entre los sistemas políticos de Chile y Argentina. Por un lado, Chile es un Estado unitario, con una administración funcional y territorialmente descentralizada o desconcentrada en conformidad a la ley (Constitución política de Chile, 1980, art. 3). No obstante, Chile es uno de los países más centralizados de América Latina en temas políticos, fiscales y administrativos (Vial, 2013; Gómez S. y Jiménez, 2011; Montecinos, 2005).

Además, Chile se divide en 16 regiones, las que a su vez se dividen en provincias, que en su interior contienen a los municipios. En total hay 345 municipios a lo largo del país. Estas unidades administrativas, a diferencia de los Estados federales, no tienen la capacidad de dictar leyes, sino que todas se rigen por la legislación que surge del gobierno central. Por lo tanto, no hay diferencias entre la legalidad de una comuna u otra y su margen de acción solo les permite hacer ordenanzas para regular la aplicación de las leyes de rango nacional (Vial, 2013).

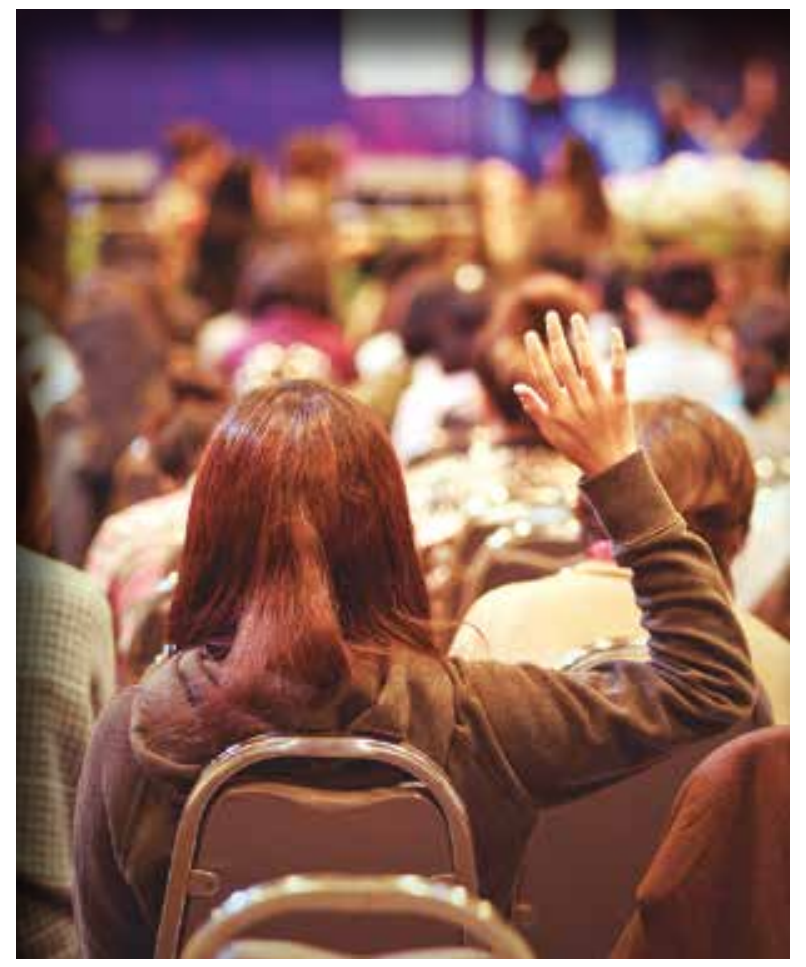


Actualmente, las municipalidades tienen diversas responsabilidades. Al respecto, Vial (2016) señala que están las que comparten con otros órganos centralizados y las de exclusiva responsabilidad. Dentro de las primeras se encuentran la mayoría de las facultades que tienen las municipalidades, lo cual las deja supeditadas a organismos centralizados. Por otro lado, en las funciones privativas destacan las de ordenamiento territorial como la elaboración del Plan Regulador Comunal (PRC), el Plan de Desarrollo Comunal (PLADECO) y las disposiciones en materias de transporte, construcción, urbanización y tránsito. Por lo tanto, excepto para estas últimas funciones, hay una alta dependencia del gobierno central, desde el punto de vista administrativo, político y fiscal (Villagrán, 2015).

Por su parte, Argentina adopta la forma representativa, republicana y federal, determinando la coexistencia de tres niveles de gobierno: el nacional, con jurisdicción en todo el territorio del país, y el 
provincial y los municipios, ambos con jurisdicción en sus respectivos territorios (Constitución de la $\mathrm{Na}$ ción Argentina, 1994). Las provincias conservan todo el poder no delegado al gobierno federal, siendo preexistentes al Estado nacional (Constitución de la Nación Argentina, 1994, art. 121). Según Cao (2007) se propone un modelo de descentralización que se justifica a partir de dos dimensiones: una política, que tiene por objetivo incrementar el poder de base territorial regional y local, y una administrativa, relacionada con la gestión desde aparatos del Estado provinciales y municipales.

En Argentina existen 24 jurisdicciones autónomas (23 provincias y la Ciudad Autónoma de Buenos Ai- res, CABA). A su vez, estas están divididas en municipios que constituyen «una comunidad natural con vida propia e intereses específicos, con necesarias relaciones de vecindad» (Iturburu, 2001: 11). A partir de los datos del Instituto de Estadísticas y Censos (INDEC), Cravacuore (2013) delimita que en Argentina existen 2.391 gobiernos locales, de los cuales 1.129 son municipalidades y 1.262 no cuentan con jerarquía municipal. El autor sostiene que el sistema municipal argentino presenta particularidades en la medida que los municipios gozan de considerable autonomía, aunque su alcance depende más de las capacidades institucionales para ejercerla que de las atribuciones asignadas por las normas legales (Cravacuore, 2013).

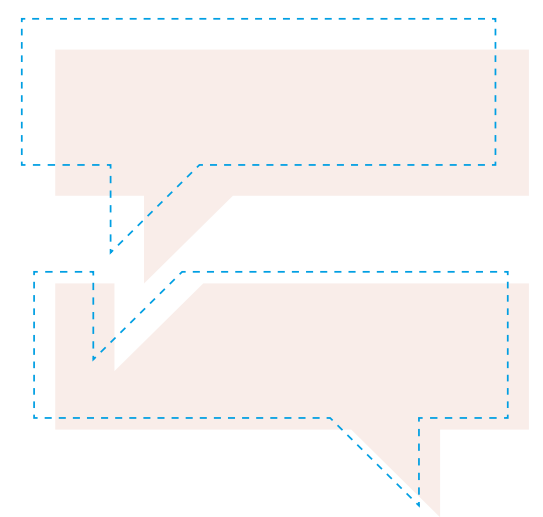




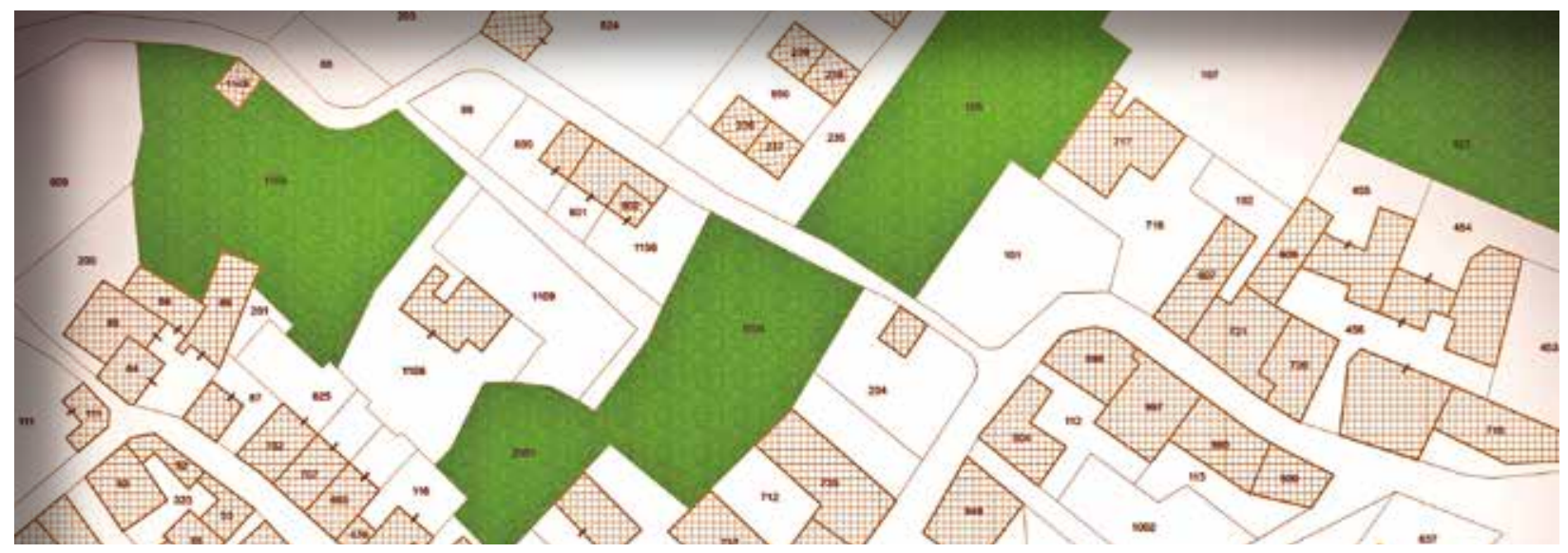

Organización del territorio en el sistema político de Chile y Argentina

\begin{tabular}{l|l|l}
\hline & CHILE & ARGENTINA \\
\hline Tipo de Estado & Unitario & Federal \\
\hline Nivel intermedio & 16 regiones & 23 provincias y la CABA \\
\hline Nivel local & 345 municipalidades & 2.391 gobiernos locales (1.129 municipalidades) \\
\hline Capacidad legislativa & Congreso nacional & Federal, provincial y municipal \\
\hline Autonomía para ordenamiento territorial & $\begin{array}{l}\text { Competencias exclusivas municipales, bajo } \\
\text { marco legal nacional }\end{array}$ & $\begin{array}{l}\text { Competencias exclusivas municipales, bajo } \\
\text { marco legal provincial y nacional }\end{array}$ \\
\hline
\end{tabular}




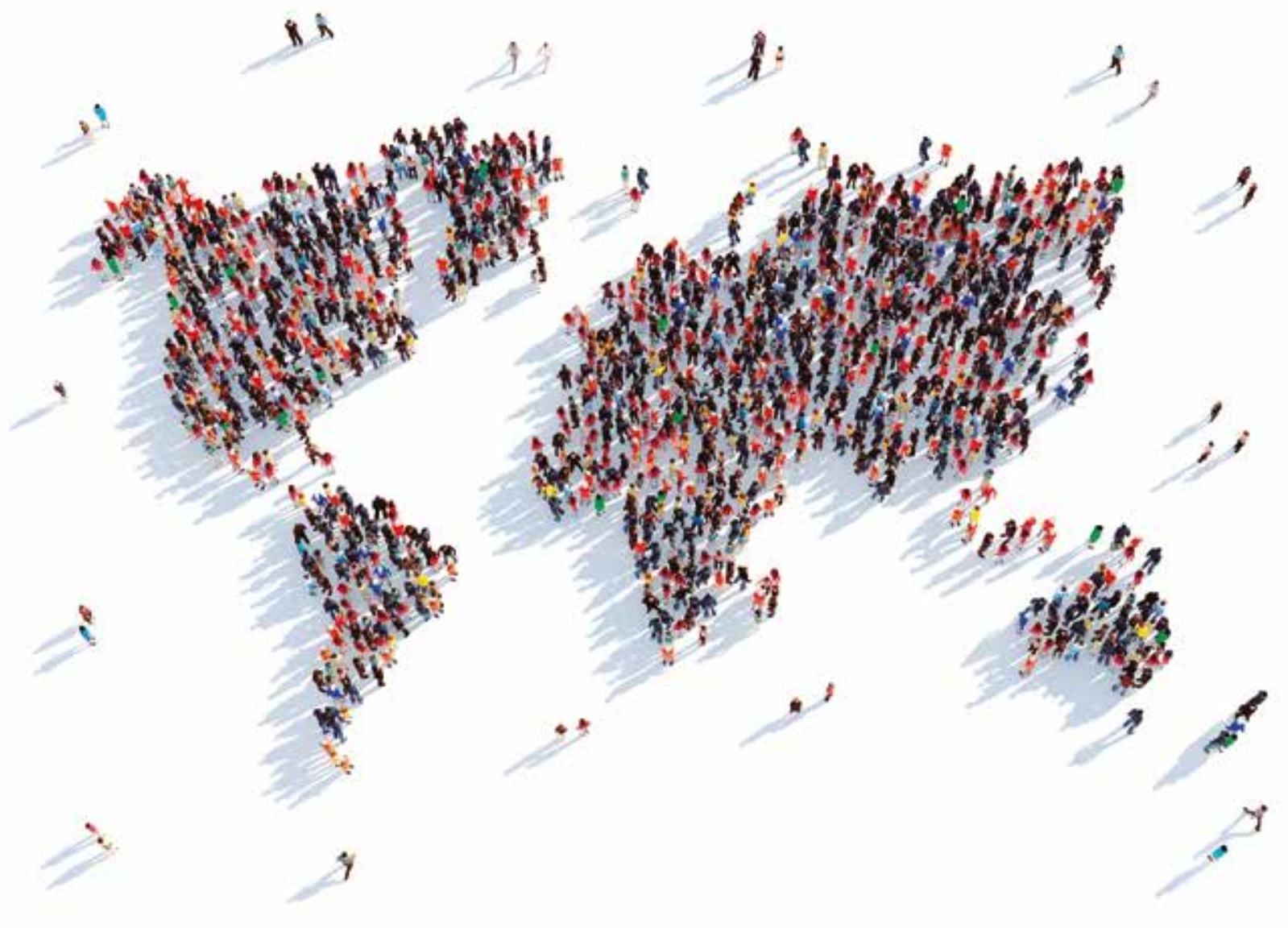




\section{Legislación sobre la planificación territorial}

Para el caso de Argentina, la legislación que se revisará será la de la Provincia de Mendoza, que se posiciona como referente en la región para el abordaje de esta problemática al ser la primera en contar con un amplio marco jurídico-normativo: la Ley de Ordenamiento Territorial y Usos del Suelo N. ${ }^{\circ} 8051 / 09$; el Plan Estratégico de Desarrollo de 2010 y el Plan Provincial de Ordenamiento Territorial (PPOT), Ley N. ${ }^{\circ}$ 8999/17. Además, la mayoría de los 18 municipios que conforman el territorio provincial cuentan con su Plan Municipal de Ordenamiento Territorial (PMOT) de acuerdo con lo dispuesto por la Ley N. ${ }^{\circ} 8051$ (Guardamagna y Reyes, 2019). En cuanto a Chile, la legislación aplica a nivel nacional y corresponde a la Ley General de Urbanismo y Construcción y su ordenanza, las que establecen los pasos y etapas necesarias para su ejecución y donde se garantiza la participación ciudadana sobre todo en la elaboración.
El proceso de elaboración de los Planes de Ordenamiento Territorial (POT) de Mendoza contempla la institucionalización de instancias participativas, tanto en el nivel provincial como en el municipal. Para ello, la ley define una secuencia de etapas en el proceso de planificación, es decir, procedimientos específicos para la elaboración de los planes, la mayoría de los cuales contemplan mecanismos de participación ciudadana.

\section{Los POT deberán permitir la intervención de todos los actores involucrados a fin de priorizar metas y objetivos, detectar problemas y conflictos y establecer las acciones a seguir, logrando el máximo nivel de beneficio público posible, definiendo propósitos, objetivos y metas compartidas, que permitan alcanzar los objetivos fijados por los planes (Inc. I. Anexo 2. Art. 11. Cap. 2. Ley N. ${ }^{\circ} 8051 / 09$ ).}

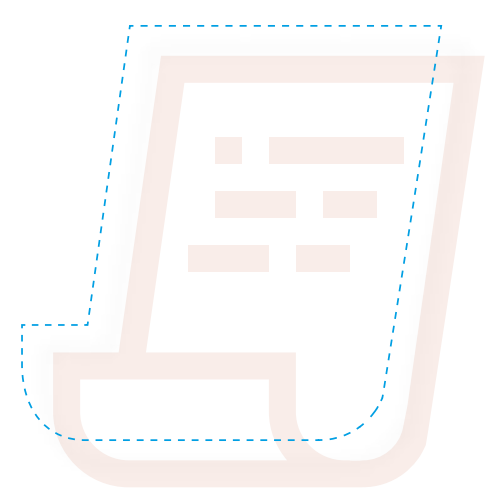


Secuencia del proceso de planificación para la elaboración de los POT
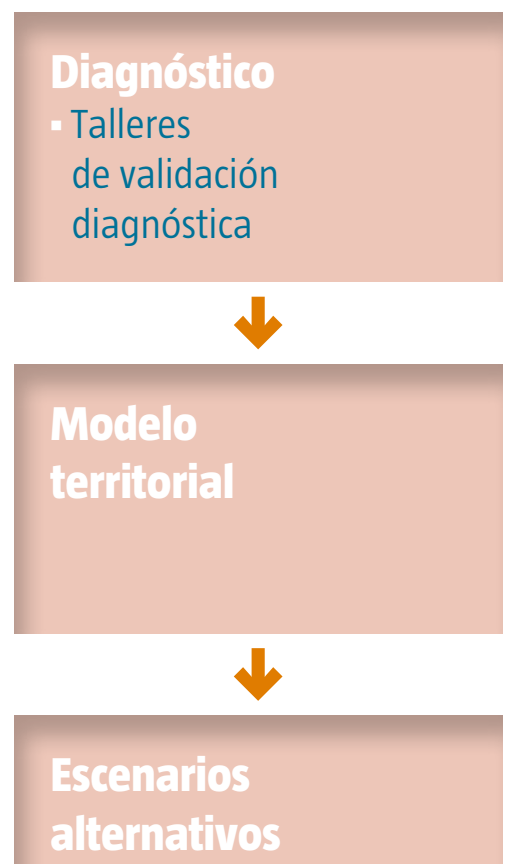
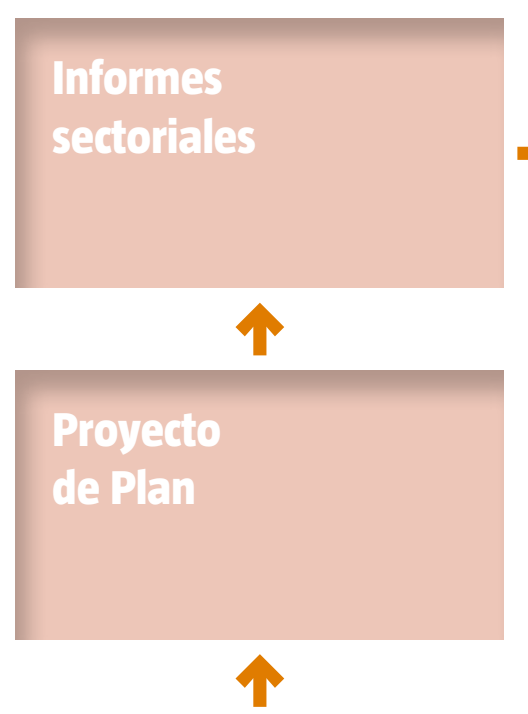

Identificación

de acciones
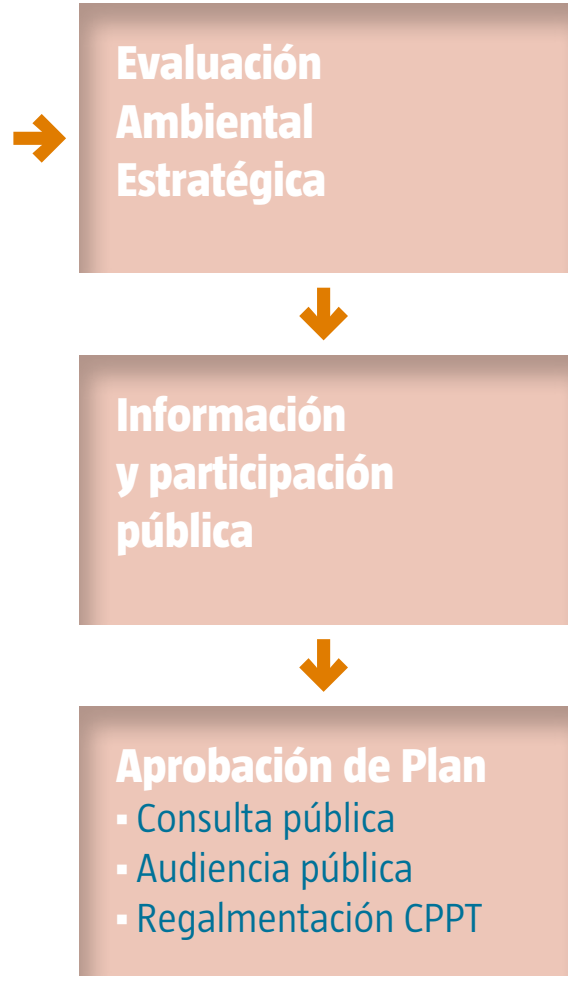

Fuente: Guardamagna (2016) 
En concreto, la ley regula dos procedimientos específicos, aunque también prevé el involucramiento ciudadano en la etapa diagnóstica. Estos son: la consulta pública y las audiencias públicas. El objetivo de la primera es «garantizar la participación y el derecho a la información mediante un procedimiento que contribuya a la toma de decisiones por parte de la autoridad de aplicación (art. 44. Ley N. ${ }^{\circ}$ 8051/09), mientras que las audiencias públicas se convocan posterior a la consulta pública para resolver temas trascendentales para la población de la provincia (art. 48. Ley N. $\left.{ }^{\circ} 8051 / 09\right)$. Además, la norma contempla la existencia de un Consejo Provincial de Ordenamiento Territorial (CPOT). Se trata de un organismo consultor y asesor, creado por el artículo 40 de la Ley N. ${ }^{\circ} 8051$ y conformado por representantes de organismos provinciales, municipios y organizaciones de la sociedad civil vinculadas a las temáticas territoriales.

A su vez, el Plan Provincial de Ordenamiento Territorial también reconoce un rol central a la participación ciudadana. Entre sus objetivos generales define: impulsar y promover los procesos de integración y coordinación entre la provincia y los municipios para lograr políticas consensuadas de desarrollo territorial, garantizando la participación ciudadana y de las organizaciones intermedias mediante mecanismos claros y transparentes de información pública y respeto por el derecho de iniciativa, propiciando la solución concertada de conflictos y diferencias (Inc. F. Ley N. ${ }^{\circ} 8999,2017$, art. 3).
En general, las etapas definidas por la norma se cumplen en lo formal, sin embargo, incluso en la elaboración del plan, hay inconsistencias teórico-metodológicas en la construcción de los diagnósticos y su posterior articulación (Guardamagna y Cueto, 2015). Guardamagna (2016) revela que existe un excesivo normativismo de la Ley $N .^{\circ} 8051$, lo que genera obstáculos entre la elaboración y la implementación del proyecto, y le resta adaptabilidad a la política, además que, a pesar de que se defina la participación ciudadana como un elemento fundamental en la política de ordenamiento territorial, esta no se ha logrado garantizar en lo formal. 
En el caso de Chile, la legislación que rige todo el proceso de diseño y modificación de Planes Reguladores Comunales (PRC) es la Ley General de Urbanismo y Construcción y su ordenanza. En aquellos documentos normativos se establece que el PRC definirá la planificación urbana comunal, que se refiere a «[...] aquella que promueve el desarrollo armónico del territorio comunal, en especial de sus centros poblados, en concordancia con las metas regionales de desarro\|lo económico-social.» (Decreto N. $\left.{ }^{\circ} 458,1976\right)$. Este instrumento, por su valor planificador de la ciudad y de la vida de la comuna, es determinante para el futuro del territorio comunal. Por lo tanto, su proceso de diseño o actualización permite elaborar el objetivo y propósito que tendrá la comuna para sus habitantes (Mlynarz Puig, 2007). Durante todo el proceso técnico de elaboración y de aprobación se contemplan instancias de participación ciudadana:

$>$ informar a la comunidad sobre las características del instrumento de planificación, $>$ realizar audiencias públicas en los barrios,

> consultar la opinión del Consejo Comunal de la Sociedad Civil (COSOC),

$>$ exponer el proyecto a la comunidad,

$>$ consultar a la comunidad por medio de una audiencia pública y,

$>$ permitir la formulación de observaciones sobre el anteproyecto (Decreto N. ${ }^{\circ} 458,1976: 20$ ).

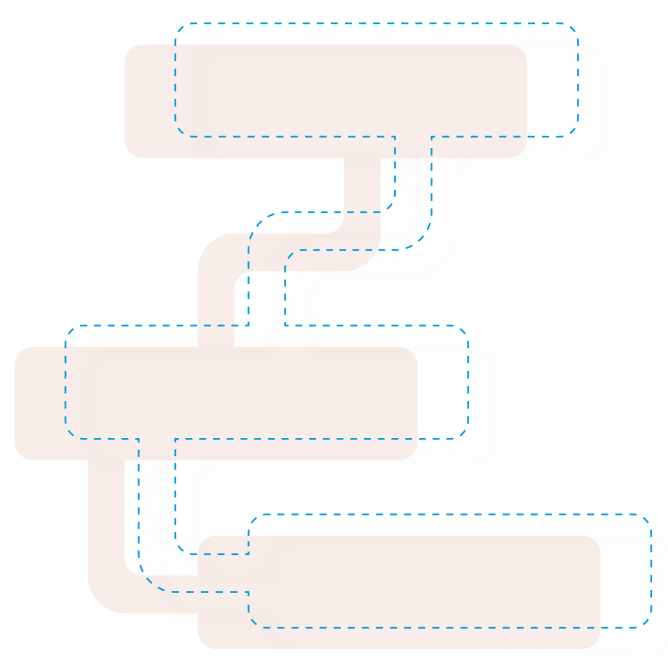


Procesos y trámites - planes reguladores comunales, Chile

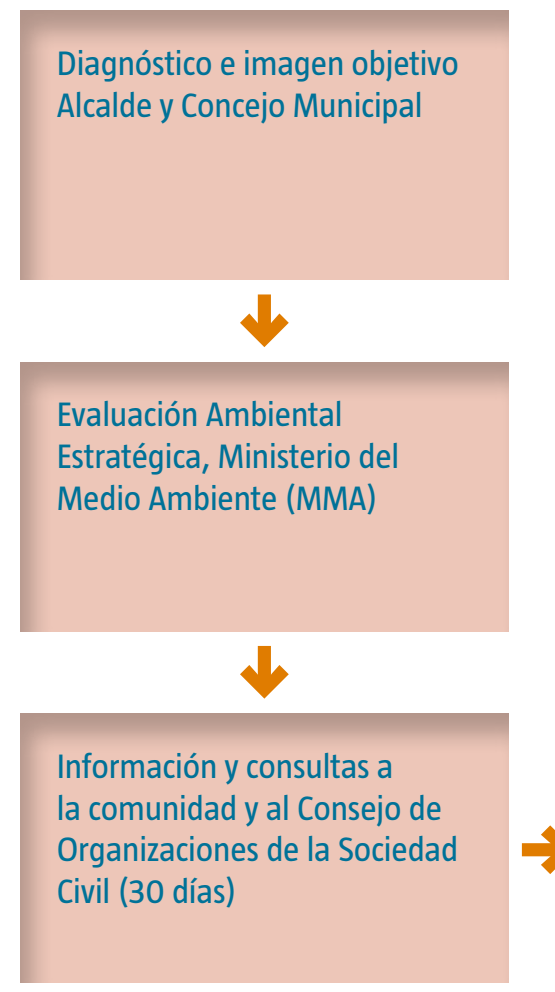

A pesar de las instancias de participación anteriormente expuestas, diferentes autores argumentan que la ciudadanía percibe que tiene poca incidencia en la toma de decisiones en política urbana y urbano/rural, lo que produce desinterés y desconfianza en los mecanismos existentes para participar (Hermann y Klave-
Promulgación, por intendente 0 alcalde, en Diario Oficial Revisión por Contraloría

Informe técnico Secretaría Regional Ministerial (Seremi) - Ministerio de Vivienda y Urbanismo (MINVU)

Revisión de comentarios y pronunciamiento oficial del Concejo Municipal (60 días)
Aviso de promulgación a Servicio de Impuestos Internos

Activar en Conservador de Bienes Raíces, división de Desarrollo Urbano y secretaría regional de MINVU y municipalidad

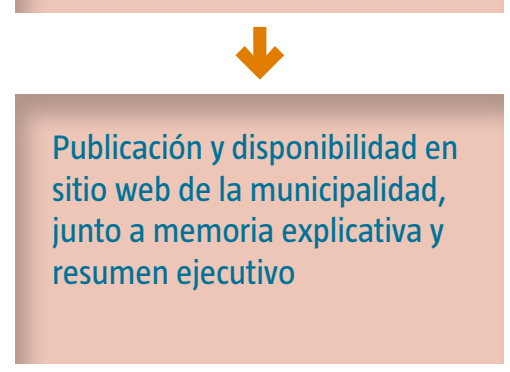

Fuente: elaboración propia ren, 2016; Poduje, 2008; Apablaza y Ruiz, 2010). En este sentido Hermann (2014) argumenta que el Plan Regulador Comunal se caracteriza más bien por una participación simbólica, donde el flujo de información es unilateral, lo cual deja a las personas sin un poder real de negociación o de decisión sobre este. 


\section{$\Rightarrow$ CONCLUSIONES}

Comenzamos este estudio preguntándonos si las dinámicas de los sistemas políticos de Argentina - federal- y Chile - centralizado - podrían impactar en el diseño, implementación y evaluación de los mecanismos de participación. Creíamos que sí.

Sin embargo, luego de años de estudiar la implementación de este tipo de procesos en ámbitos locales de gobierno, donde la proximidad Estado-ciudadanía-Estado es más tangible, y más aún en el marco de políticas eminentemente participativas, como son las de desarrollo territorial, entendemos que la centralización o descentralización no necesariamente es una variable determinante en el resultado de estos.

Nuestras indagaciones nos indican que:

$>$ En ambos países los marcos jurídico-normativos reconocen la necesidad de participación en las políticas de desarrollo territorial y definen mecanismos participativos para los distintos momentos de esta. 
En ambos países los gobiernos y administraciones locales cuentan con autonomía en cuanto al diseño de sus planes de ordenamiento territorial en el marco de las leyes nacionales (Chile) o provinciales (Mendoza).

$\rightarrow$ En ambos países dichos instrumentos participativos son entendidos como en exceso formales $y$ consultivos, sin mayor incidencia en la toma de decisiones, ni en el seguimiento y evaluación de las políticas orientadas al desarrollo de los territorios.

Estas apreciaciones preliminares nos permiten inferir que los marcos normativos son necesarios, pero no suficientes a la hora de propiciar políticas públicas participativas, aun cuando las gestiones gubernamentales tengan la autonomía para hacerlo. En todo caso, cuestiones más coyunturales, como la voluntad política y la discrecionalidad de las autoridades locales, son algunos de los elementos que, al parecer, terminan influyendo en la posibilidad de garantizar un involucramiento real, plural, equitativo y sostenido en el tiempo de la ciudadanía.

A simple vista, a pesar de tener estructuras distintas, los mecanismos de participación revisados tienen carencias en cuanto a su incidencia y evaluación. Es necesario, entonces, desentrañar cuáles son las dimensiones propias de la gestión territorial que mayor incidencia tienen en la implementación de mecanismos de participación ciudadana, principalmente en ámbitos locales de gobierno. Para ello, el estudio comparado de casos locales en América Latina nos podrá alumbrar en torno a los alcances de la participación ciudadana en un tema tan primordial como el desarrollo territorial. 


\section{$\rightarrow$ BIBLIOGRAFÍA}

$>$ Alburquerque, F. (2004).

"Desarrollo económico territorial. Guía para agentes». Instituto de Desarrollo Regional.

Sevilla: Fundación Universitaria.

$>$ Andrews, R., Cowell, R., y Downe, J. (2008).

"Support for active citizenship and public service performance: an empirical analysis of English local authorities». Policy \& Politics, 36(2): 225-243. https://doi.org/10.1332/030557308783995044

$>$ Cao, H. y Vaca, J. (2007).

«El fracaso de la descentralización Argentina Andamios». Revista de Investigación Social, 4(7): 249-267.

México: Universidad Autónoma de la Ciudad de México Distrito Federal.

$>$ Cooper, M.; Henríquez, C. (2010).

«Planificación territorial y crecimiento urbano: desarticulaciones y desafíos de la sostenibilidad urbano-regional en Santiago metropolitano». Scripta Nova, Revista Electrónica de Geografía y Ciencias Sociales, 14. Disponible en https://bit.ly/2JP49nP

$>$ Cravacuore, D. (2013).

Gobiernos locales en Argentina. Argentina: Universidad Nacional de Quilmes.

$>$ De Sousa Santos, B. (2003).

Democracia y Participación: el ejemplo del presupuesto participativo de Porto Alegre.

Barcelona: El Viejo Topo.

$>$ Evans, A.; Campos, A. (2012).

"Open Government Initiatives: Challenges of Citizen Participation». Journal of Policy Analysis and Management. 32(1): 172-185. 
> Gómez S., J. C. y Jiménez, J. P. (2011).

El financiamiento de los gobiernos subnacionales en América Latina: un análisis de casos:

CEPAL (serie macroeconomía del desarrollo), 111. Disponible en https://bit.ly/3boKvkU

\section{$>$ Guardamagna, María Melina (2016).}

"El ordenamiento del territorio en Mendoza, Argentina: la implementación iparticipativa? de una política». Revista de Geografia e Ordenamento do Território, GOT. 10: 159-187.

\section{$>$ Guardamagna, María Melina y Cueto, Walter (2015).}

«La implementación de la Política de Ordenamiento Territorial en Mendoza, Argentina: una mirada crítica sobre diseño del Plan Provincial». Revista Enfoques. 13(22): 135-153.

\section{$>$ Guardamagna, María Melina y Reyes, Malena Lucía (2019).}

"El desafío de ordenar el territorio en democracia iretórica participativa o involucramiento real de la ciudadanía?», Economía, Sociedad y Territorio, 18: 1003 - 1033.

> Harrison, T.M. y Sayogo, D.S. (2014).

"Transparency, participation, and accountability practices in open government: a comparative study». Government Information Quarterly, 41: 513-525.

$>$ Herrmann, G. (2014).

"Hacer Ciudad: Problemas y desafíos de la participación ciudadana en la planificación territorial de Santiago". Revista 180, 34. 
$>$ Herrmann, G.; van Klaveren, A. (2016).

"Disminución de la participación de la población en organizaciones sociales durante los últimos trece años en Chile e implicaciones para la construcción de una política de planificación urbana más participativa». EURE 42(125): 175-203. https://dx.doi.org/10.4067/S0250-71612016000100008.

$>$ Irvin, R. A., y Stansbury, J. (2004).

"Citizen Participation in Decision Making: ¿Is It Worth the Effort?» Public Administration Review, 64(1): 55-65. https://doi.org/10.1111/.17540-6210.2004.00346.x

$>$ Iturburu, M. (2001).

Potestades y Restricciones Constitucionales para un Nuevo Modelo de Gestión Local. Instituto Nacional de la Administración Pública, INAP. Municipios Argentinos. $2^{\circ}$ edición. Dirección Nacional de Estudios y Documentación.

$>$ Manero, M. (2010).

"La participación ciudadana en la ordenación del territorio: posibilidades y limitaciones». Revista Cuadernos Geográficos, 47(2): 47-71.

$>$ Mlynarz, D. (2007).

Participación ciudadana en el proceso de diseño y modificación de los planes reguladores comunales: propuesta desde diversos actores. Maestría en Política y Gobierno, FLACSO sede Chile. Santiago. Disponible en https://bit.ly/2JUc3fu

$>$ Montecinos, E. (2005).

"Antecedentes sobre la relación histórica centralismo y descentralización en Chile». Revista Venezolana de Gerencia, 10(31). Disponible en https://bit.Iy/2wFP1pY 
Patsias, C., Latendresse, A., y Bherer, L. (2013).

"Participatory democracy, decentralization and local governance: The montreal participatory budget

in the light of "empowered participatory governance"». International Journal of Urban and Regional Research, 37(6): 2214-2230. https://doi.org/10.1111/j.1468-2427.2012.01171.x

Poduje, I. (2008).

Participación ciudadana en proyectos de infraestructura y planes reguladores. Santiago: Dirección de Asuntos Públicos UC. Disponible en https://bit.ly/2V9gABz

$>$ Pragier, D. (2009).

"La participación ciudadana en la bibliografía de desarrollo local. Algunas consideraciones sobre la bibliografía, contexto de surgimiento y enfoques». Ponencia presentada en el XXVII Congreso de la Asociación Latinoamericana de Sociología. VIII Jornadas de Sociología de la Universidad de Buenos Aires. Asociación Latinoamericana de Sociología, Buenos Aires.

> Schejtman, A. y Berdegué, J. (2004).

"Desarrollo Territorial Rural». Debates y temas rurales (1), RIMISP. Santiago de Chile:

Centro Latinoamericano para el Desarrollo Rural. Disponible en https://bit.ly/34otLBC

$>$ Sili, M. (2005).

La Argentina rural. De la crisis de la modernización agraria a la construcción de un nuevo paradigma de desarrollo de los territorios rurales. Buenos Aires: INTA Ediciones. 
$>$ Vial, M. C. (2013).

"Political and fiscal and fiscal decentralization in South America: a comparative analysis of Bolivia, Chile, Colombia, Ecuador and Perú». In J. R. Cuadrado-Roura y P. Aroca (Eds.), Regional problems and policies in Latin America. Berlín: Springer-Verlag.

\section{-- (2016).}

"Las Municipalidades en Chile». En M. C. Vial y J. M. Ruano, Manual de Gobiernos locales en Iberoamérica. Santiago: Universidad Autónoma de Chile. Disponible en https://bit.ly/3elTbEc

\section{$>$ Villagrán, M. (2015).}

Manual de Derecho Municipal. Santiago: RIL Editores.

\section{$>$ Zoido, F. (2007).}

"Territorialidad y gobierno del territorio, hacia una nueva cultura política». En Farinos, J. y Romero, J., Territorialidad y buen gobierno para el desarrollo sostenible. Nuevos principios y nuevas políticas en el espacio europeo. Valencia: Universidad de Valencia.

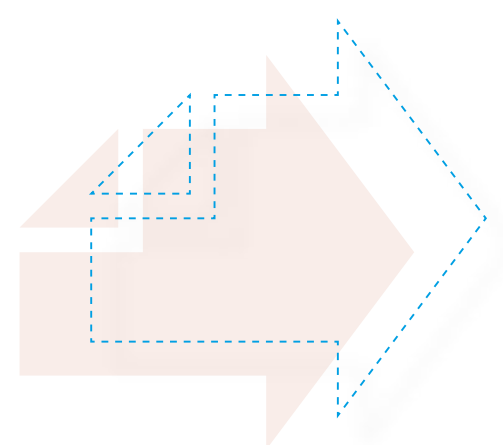




\section{Normas consultadas}

\section{ARGENTINA}

\section{Argentina (1994).}

Constitución Nacional, Buenos Aires. Disponible en https://bit.ly/2wCuJh5

Provincia de Mendoza. Ley N. ${ }^{\circ} 8051 / 09$.

Ley de Ordenamiento Territorial y usos del suelo. Mendoza. 2009. Disponible en: https://bit.ly/3b1/4yl

Provincia de Mendoza. Ley N. ${ }^{\circ} 8999 / 17$.

Plan Provincial de Ordenamiento Territorial. Mendoza, Argentina. 2017. Disponible en https://bit.ly/2V2Z3L8

\section{CHILE}

Constitución Política de la República de Chile (17 de septiembre de 1980).

Diario Oficial. Ministerio Secretaría General de la Presidencia. Disponible en: http://bcn.cl/1uva9

Decreto N. ${ }^{\circ} 458$ (13 de abril de 1976,).

Ley general de urbanismo y construcciones. Santiago: Diario Oficial de la República de Chile.

Disponible en http://bcn.cl/1uuhi

Ley N. ${ }^{\circ} 18.695$ (9 de mayo de 2006).

Ley orgánica Constitucional de Municipalidades. Santiago: Diario Oficial de la República de Chile. Disponible en http://bcn.cl/7uwcv

Ley N. ${ }^{\circ} 19.300$ (9 de marzo de 1994).

Ley sobre bases generales del medio ambiente. Santiago: Diario Oficial de la República de Chile. Disponible en http://bcn.cl/7ux38 


\section{Melina Guardamagna}

Doctora en Ciencia Política. Investigadora del Consejo Nacional de Investigaciones Científicas y Técnicas (CONICET), Argentina. Directora del Centro de Estudios de Innovación Institucional (CEII) de la Universidad Nacional de Cuyo y directora ejecutiva de la Red Interamericana de Educación en Administración Pública (INPAE).

\section{José Hernández Bonivento}

Doctor en Ciencias Políticas y de la Administración por la Universidad Complutense de Madrid (España). Director del Instituto Chileno de Estudios Municipales (ICHEM) de la Universidad Autónoma de Chile.

\section{¿CÓMO REFERENCIAR?}

Guardamagna, M.; Hernández, J. (2020). Mecanismos de participación ciudadana para el desarrollo territorial. Una agenda de investigación comparada. Estudio corto ICHEM N. ${ }^{\circ}$ 5. Santiago de Chile: Universidad Autónoma de Chile.

Disponible en: http://ichem.uautonoma.cl/category/publicaciones/

Otros títulos de esta serie:

$\rightarrow$ Transparencia en las municipalidades.

Una mirada a 10 años de la ley 20.285

Descárgalo en: https://bit.ly/2QlLbdw

$\rightarrow$ Barreras para la organización popular en el Chile democrático Descárgalo en: https://bit.ly/2M9VIGZ

$>$ Políticas y programas de infancias y adolescencias a nivel municipal Descárgalo en: https://bit.ly/2rzB9uY

$>$ Sistema de Certificación Ambiental Municipal. Una breve evaluación a diez años de su implementación Descárgalo en: https://bit.ly/2yOtegy 